\title{
Analysis and research on energy utilization efficiency of port portal crane
}

\author{
Leilei $\mathrm{Liu}^{1 *}$, Kun $\mathrm{Guan}^{2}$, and Peisen $\mathrm{Wu}^{1}$ \\ ${ }^{1}$ Tianjin Research Institute for Water Transport Engineering, Key Laboratory of Environmental \\ Protection Technology on Water Transport, Ministry of Transport, Tianjin, China \\ ${ }^{2}$ Tianjin Port Zhongmei Huaneng Coal Terminal Co., Ltd
}

\begin{abstract}
Portal crane is one of the important equipment of bulk terminal, which is specially used for shore loading and unloading operation. It mainly consumes electric energy. In order to clarify the utilization of electric energy during the operation of portal crane and analyze the energy utilization efficiency, a portal crane of a bulk terminal was selected as the research object and the energy utilization was tested. The voltage, current, imbalance, harmonic, power and energy efficiency of portal crane were analyzed in his paper. Some suggestions were put forward to improve the energy utilization efficiency and reduce energy consumption of portal crane.
\end{abstract}

Keywords: Port; Portal crane; Energy utilization efficiency; Harmonic.

\section{Introduction}

Portal crane is one of the main equipment for port shore loading and unloading ${ }^{[1]}$, and also one of the main energy consumption equipment for port production. Therefore, improving the energy utilization efficiency of portal crane through technical and management means plays a significant role in reducing the production energy consumption of bulk terminal. The research on crane energy consumption mainly focuses on the detection of its working state and system. Haibin Yang ${ }^{[2]}$ designed the crane state monitoring system. Hongwei Liu ${ }^{[3]}$ remotely monitored the crane operation state information through different strategies. Bin $\mathrm{Chen}^{[4]}$ designed the data warehouse system as the platform to analyze the performance trend of the crane. According to the requirements of "The method of checking and measuring energy utilization efficiency for port electric-crane" ${ }^{\prime[5]}$, this paper monitors and analyzes the energy consumption of portal crane during operation, finds out the shortcomings in energy utilization, and puts forward targeted improvement schemes, which plays an important role in reducing port operation energy consumption.

\section{Design of energy efficiency test scheme}

\subsection{Testing standards and methods}

*Corresponding author: eca@foxmail.com 
The energy efficiency test shall be carried out in accordance with the requirements of "The method of checking and measuring energy utilization efficiency for port electric-crane" (JTT 314-2009).

The test steps are as follows:

(1) Lift the load at the rated speed. The load mass is $80 \%$ of the rated lifting capacity. The amplitude is $50 \%$ of the maximum amplitude. And the load lifting height is $10 \mathrm{~m}$. Place the load to the lifting position.

(2) The load mass is $80 \%$ of the rated lifting capacity. The rotation radius is $50 \%$ of the maximum load. Rotate $180^{\circ}$ clockwise. After the load is stable, rotate $180^{\circ}$ counterclockwise.

(3) The load mass is $80 \%$ of the rated lifting capacity. The amplitude changes from $50 \%$ of the maximum amplitude to the maximum amplitude. After the load is stable, return to the position before luffing.

(4) The lifting mechanism rises and falls by $10 \mathrm{~m}$ at the rated speed, and the amplitude is $50 \%$ of the maximum amplitude.

\subsection{Testing process}

No-load lifting $\rightarrow$ No-load luffing (smaller) $\rightarrow$ No-load rotation $\rightarrow$ No-load luffing (larger) $\rightarrow$ No-load lowering $\rightarrow$ Grasp the load $\rightarrow$ Loaded lifting $\rightarrow$ Loaded luffing (smaller) $\rightarrow$ Loaded rotation $\rightarrow$ Loaded luffing (larger) $\rightarrow$ Loaded lowering $\rightarrow$ Load lowering $\rightarrow$ Noload lifting $\rightarrow$ No-load luffing (smaller) $\rightarrow$ No-load rotation $\rightarrow$ No-load luffing (larger).

\section{Voltage and current test and analysis}

In this measurement, several working cycles are measured, and the extreme values of voltage and current are shown in Table 1.

Table 1. Voltage and current extremum.

\begin{tabular}{|c|c|c|c|c|c|}
\hline No. & Test content & L1 & L2 & L3 & $\begin{array}{c}\text { Average } \\
\text { value }\end{array}$ \\
\hline 1 & $\begin{array}{c}\text { Maximum } \\
\text { voltage(V) }\end{array}$ & 411.45 & 413.1 & 411.65 & 412.1 \\
\hline 2 & $\begin{array}{c}\text { Minimum } \\
\text { voltage(V) }\end{array}$ & 356.65 & 358.15 & 360.25 & 358.35 \\
\hline 3 & $\begin{array}{c}\text { Maximum } \\
\text { current(A) }\end{array}$ & 572 & 570 & 578 & 573.3 \\
\hline 4 & $\begin{array}{c}\text { Minimum } \\
\text { current(A) }\end{array}$ & 7 & 1 & 7 & 5 \\
\hline
\end{tabular}

In this workflow, during heavy load lifting, the voltage decreases, the current increases greatly, the peak value reaches more than $560 \mathrm{~A}$, the power factor is 0.99 , and the electric energy is converted into gravitational potential energy and kinetic energy. During the intermittent period of loading, the voltage is stable, the current value is the smallest, and it is basically standby loss. When the heavy load drops, the gravitational potential energy is released and converted into electric energy and kinetic energy. The whole process is in a cycle, the current changes periodically, and the voltage decreases when the current increases greatly. At this time, the voltage drop is mainly caused by heavy load startup. The maximum power supply voltage is $413.1 \mathrm{~V}$ and the minimum voltage is $356.65 \mathrm{~V}$. The power supply voltage of portal crane is $380 \mathrm{~V}$. The voltage deviation is $6.14 \%-8.71 \%$, and $95 \%$ of the voltage is distributed within $408.4 \mathrm{~V}$, as shown in Figure $1.95 \%$ of the current is distributed within 309 A, as shown in Figure 2. 


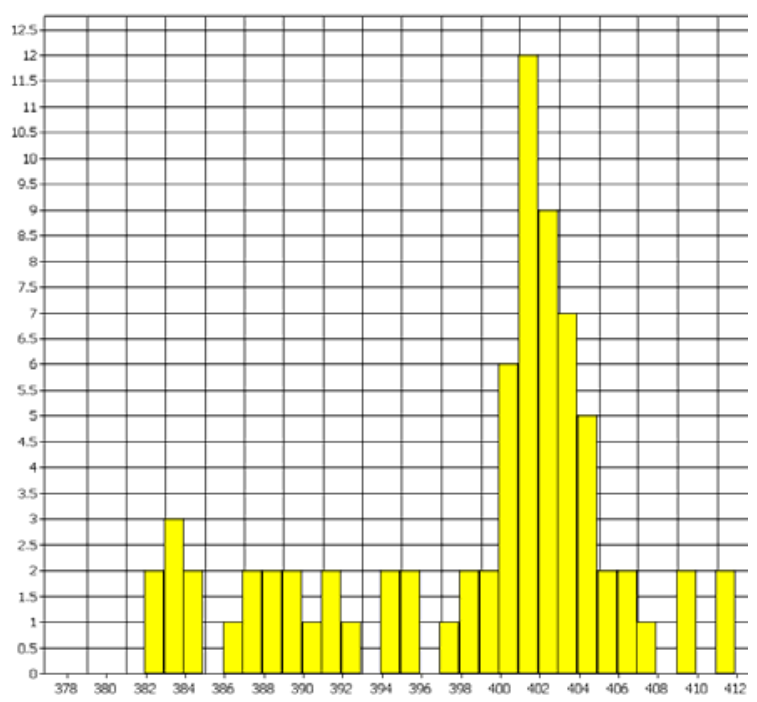

Fig. 1. Probability diagram of voltage distribution in normal period.

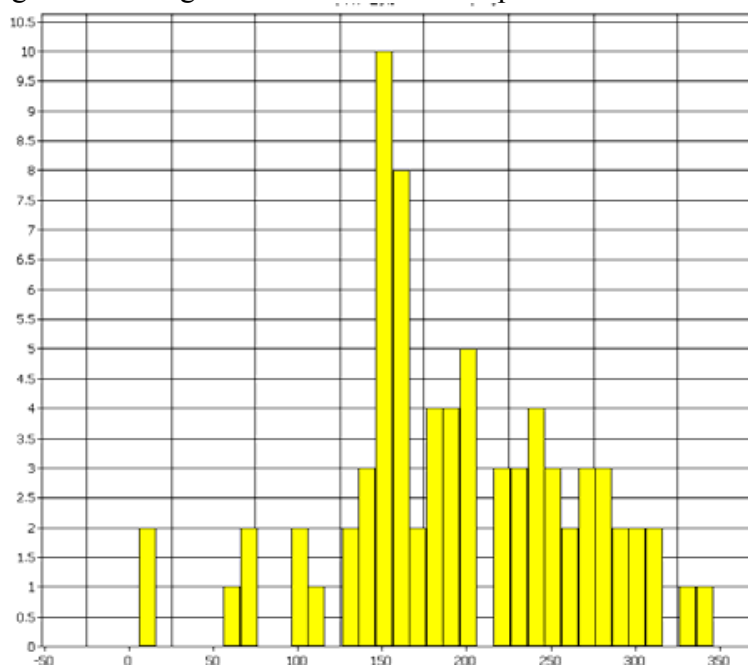

Fig. 2. Probability diagram of current distribution in normal period.

\section{Imbalance test and analysis}

The imbalance of supply voltage is within $0.65 \%$, less than $2 \%$ of the standard value. The current imbalance is basically kept within $15 \%$. The situation of large imbalance value is mainly that the deceleration time and current drop after the lifting weight reaches the required height, and the current difference is small, but when it accounts for a large proportion of the current value, the current imbalance is not more than $5 \%$ in the main lifting and rotation process.

The included angle of voltage and current of each phase changes greatly, basically between 0-60 degrees. The reactive power fluctuates greatly, the power factor changes greatly, and the electric energy loss caused by reactive power is large. Especially in the case of no-load, rotating forward and descending with load, according to the working mechanism, except that the power factor of main load lifting is very high, which is between 
0.97-0.99, the power factor is between $0.44-0.85$ at other times, which is negative when falling. In standby, the power factor is about 0.45 .

$95 \%$ of voltage imbalance is within $0.3 \%$, and $95 \%$ of current imbalance is within $15 \%$. Voltage imbalance is better than current imbalance. The possible causes of voltage imbalance are high-order harmonic and reactive power, but the proportion is small and does not affect the actual operation.

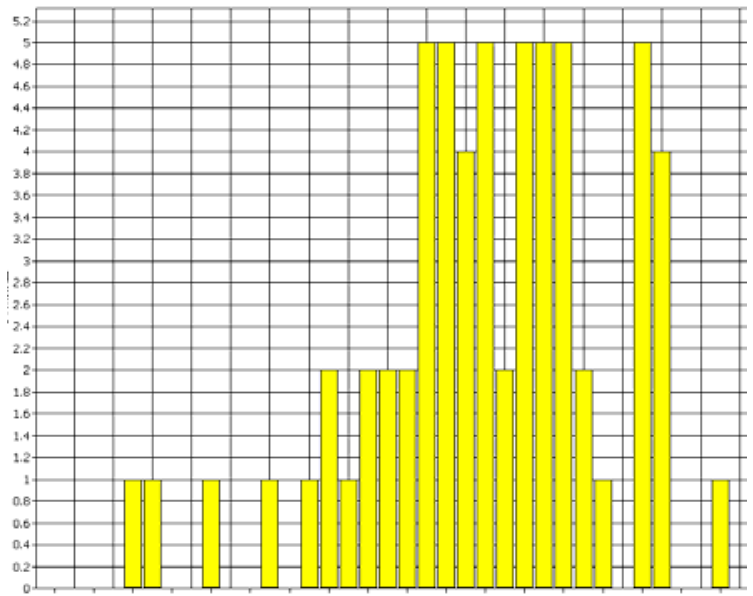

Fig.3. Voltage imbalance distribution.

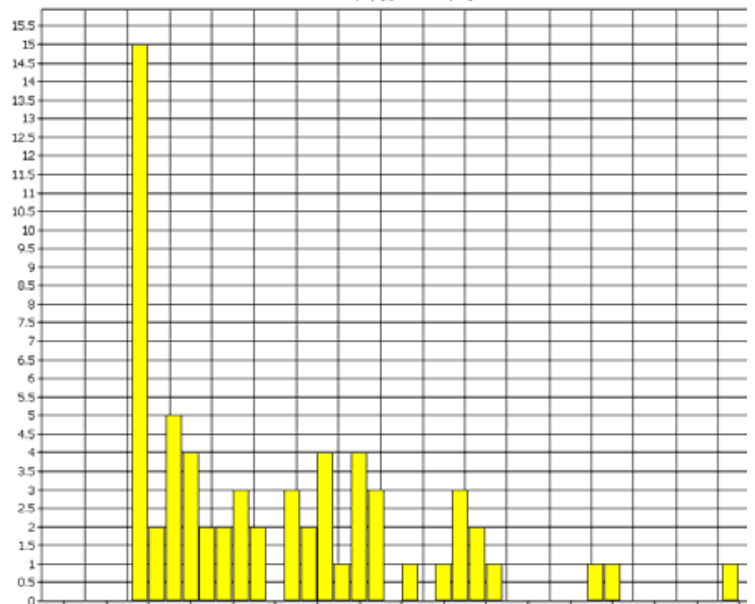

Fig.4. Current imbalance distribution.

\section{Power test analysis}

The portal crane works periodically. During heavy load lifting, the power consumption is the largest, and the instantaneous power is large. During heavy load falling, the gravity potential energy is mainly in the power generation state, and the instantaneous power is negative.

It can be found that there are positive work and negative work (feedback electric energy) in portal crane. Because the voltage and frequency of feedback electric energy do not match with the power grid, it will not only affect the measurement of electric energy, but also worsen the power quality of the power grid. At the same time, due to the decline of power quality during the operation of portal crane, the feedback power quality is not high, which affects itself or other electrical equipment, such as reducing the service life of lighting 
equipment. If the feedback electric energy can be effectively collected or reasonably utilized, it will save a lot of electric energy and be conducive to the use of equipment.

The frequency and voltage imbalance are acceptable, while the average power factor is only 0.31 . If negative power is not considered, the average power factor is about 0.7 , which is relatively low in multiple working cycles. The power factor is high only when it is raised, but the weight in the whole working cycle is low, so the average value is also low.

In a working cycle, the power consumption of bulk cargo handling is basically maintained at $2-3 \mathrm{kWh}$. The loading and unloading are discontinuous. The longer the intermittent period is, the longer the standby time is. The greater the percentage of standby power consumption in each working cycle is, and the more power consumption per ton of cargo is.In this workflow, the lifting operation is the main power consumption stage, with high power factor and fixed power consumption. Other working links have low power factor and large line loss. Slow operation speed will increase the overall power consumption of the working cycle, so appropriately increasing the working speed other than lifting will help to reduce the loss.

\section{Conclusions and recommendations}

(1) The portal crane generally operates discontinuously. Reducing standby time and increasing work compactness are helpful to improve work efficiency and reduce energy consumption. It is suggested to mainly adopt optimal scheduling to make the process connection closer and reduce the equipment empty consumption in priority to the technical transformation.

(2) When the equipment works for a long time, the average power factor is not high. It can be considered to add dynamic reactive power compensation device in the nearby power transformation and distribution room to reduce reactive power loss and improve power factor.

(3) The working condition of the bulk cargo terminal is poor. It is recommended to regularly remove dust, fixing screws and other maintenance work of the electric control equipment to reduce the failure rate of the equipment and improve the continuous working time of the equipment while increasing the stability of the equipment.

\section{Acknowledgment}

Fund Project, Central-level nonprofit scientific research institutes basic research project special funds TKS20200205.

\section{References}

1. Xueyun Li, Jun Li. Analysis of on-line monitoring method for port gantry crane [J]. Shanghai Machine Tool, 2017, (1):11-13.

2. Haibin Yang. Design and implementation of intelligent terminal for remote condition monitoring system of large crawler crane [D]. Shanghai Jiaotong University, 2009

3. Hongwei Liu. Research and development of remote monitoring system for special crane in shipyard [D]. Tongji University, 2010

4. Bin Chen. Research and implementation of data optimization of large crane remote monitoring system [D]. Shanghai Jiaotong University, 2009

5. JTT 314-2009. The method of checking and measuring energy utilization efficiency for port electric-crane [S].Beijing: China Communications Press, 2010 